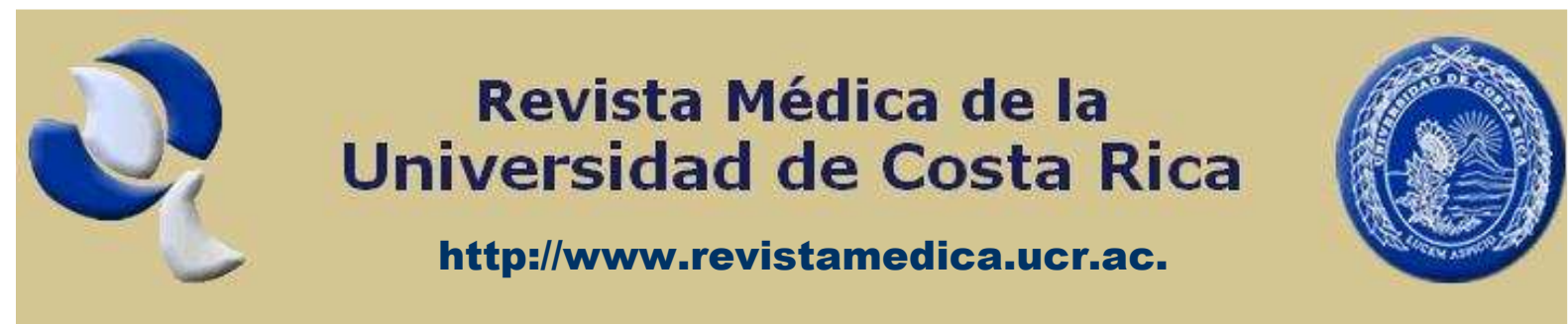

Contribución en Docencia

\title{
USO DE ANIMALES EN DOCENCIA; PERCEPCIÓN DE LOS ESTUDIANTES
}

Granados Zúñiga, Jorge

Departamento de Fisiología. Escuela de Medicina, Universidad de Costa Rica. San Pedro, Costa Rica.

\begin{abstract}
Resumen
El empleo de animales en la enseñanza de la medicina es necesario pero su uso adecuado depende en gran parte de la buena disposición de los estudiantes. Debido a la falta de información documentada sobre la percepción los estudiantes de medicina del uso de tal recurso el objetivo del presente estudio fue cuantificar esta percepción en un grupo de estudiantes de la carrera de medicina en la Universidad de Costa Rica.

Se empleó una encuesta sobre información personal del estudiante, experiencia o información previa sobre el uso de animales y conocimiento sobre la disposición final de los animales.

Al momento de la consulta un $98 \%$ de los encuestados $(n=88)$ ya habían usado animales en alguna actividad académica en la Universidad. La mayoría de los consultados $(73 \%)$ conocía de la existencia de regulaciones sobre el uso de animales; muy pocos $(36 \%)$ sabían cuál era la procedencia de los animales que utilizaban o qué destino tendrían luego de ser empleados (2\%). Aunque un $89 \%$ opinó que esta práctica aporta un beneficio en su aprendizaje también consideró tal práctica carente de alguna ventaja o francamente desventajosa. Se recomienda el uso de métodos alternativos como sustituto o complemento al empleo de animales en docencia.
\end{abstract}

Palabras claves: Animales, docencia, medicina.

Recibido: Septiembre 2007. Aprobado: Octubre 2007. Publicado: Marzo 2008.

Revista electrónica publicada por el Departamento de Farmacología de la Escuela de Medicina de la Universidad de Costa Rica, 2060 San José, Costa Rica. ${ }^{\circledR}$ All rights reserved. 


\begin{abstract}
The still necessary use of animals in physiology instruction requires an adequate attitude by students. Until now there's no information on the way medicine students perceive the use of animals in their courses. The main objective of this study was to measure that perception on a group of medicine students from the Universidad de Costa Rica.

A questionnaire on students' personal information, their expertise or previous information on use of animals and their knowledge on final disposition of animals was applied.

At moment of enquiry $98 \%$ of students $(n=88)$ already had used animals in some academic activity; despite $73 \%$ of them knew the existence of regulations on the use of animals only between $2-36 \%$ knew where the animals came from or where did they go after they were used. Finally, regardless $89 \%$ of the students thought that the use of animals provides some benefit to their learning they also saw such a practice with no advantages or with disadvantages. It is recommended the use of alternative methods as substitution or complement of animals in physiology teaching.
\end{abstract}

Key words: Animals, teaching, medicine.

\section{Introduction}

El aprendizaje adecuado requiere un procesamiento activo de la información [1]. Para ello se ha impulsado el "aprendizaje con sentido" de la fisiología propiciando que el estudiante prediga la respuesta fisiológica en el sistema si éste se altera [2].

Parte de las actividades basadas en experimentos incluyen aquellas que no requieren el uso de tejidos vivos tales como las simulaciones por computadora [ver referencias 3 y 4] y los modelos físicos; y las que sí los requieren, por ejemplo, sujetos humanos, animales o plantas [1]. Se ha señalado que las prácticas de laboratorio con animales deben ofrecerse por razones válidas cuando contribuyan con la construcción del conocimiento, habilidades y aptitudes asegurándose que los estudiantes estén apropiadamente preparados para realizar una experiencia humanitaria [5].

Por otro lado, se ha observado que las preferencias cognitivas de los estudiantes pueden afectar su desempeño en diferentes áreas temáticas [citado por 6]. Durante la enseñanza de las ciencias básicas en la carrera de medicina algunas veces se requiere utilizar animales y al hacer esto el docente supone que los estudiantes coinciden con él en la necesidad de tales prácticas así como en el valor que tienen las mismas. Sin embargo, algunos han expresado una preferencia hacia el uso de simulaciones por computadora porque objetan en principio el uso de animales o por que los abruma la experiencia [7].

En la Universidad de Costa Rica no existen estudios acerca de las preferencias de los estudiantes hacia el uso de animales dentro de los cursos de laboratorio de fisiología ni respecto a cómo perciben esta práctica. Por estas razones, el objetivo de esta investigación fue cuantificar la percepción que tienen los estudiantes que inician la carrera de medicina en la

Revista electrónica publicada por el Departamento de Farmacología de la Escuela de Medicina de la Universidad de Costa Rica, 2060 San José, Costa Rica. ${ }^{\circledR}$ All rights reserved. 
uso de animales en la docencia.

\section{Método y Material}

Se estudió una muestra de 88 estudiantes, tanto hombres como mujeres, de segundo año de medicina de la Universidad de Costa Rica. Previamente a la realización de un laboratorio del curso de Fisiología

Humana en el que se emplearían animales de laboratorio se aplicó a los estudiantes, divididos en dos grupos en días sucesivos ( 8 y 9 de noviembre de 2006), un cuestionario preparado por el autor con carácter anónimo.

\section{Resultados:}

El $48 \% \quad(n=42)$ de los estudiantes encuestados fue de género masculino y el $52 \%$ de género femenino, la edad promedio fue de 20.2 años $(S D=1.7)$ y en un $99 \%$ de los casos cursaban el segundo año de la carrera al momento de la aplicación de la encuesta. Finalmente, con respecto a la utilidad del uso de animales con fines

\section{Discusión}

Aunque un $73 \%$ de los estudiantes encuestados dice conocer las normas respecto al uso de animales muy pocos saben cuál es la procedencia de los mismos o cómo se dispone finalmente de ellos (36\% y $2 \%$ respectivamente). Debería proporcionarse a los estudiantes información precisa [8] que le ayude a comprender el valor de este reactivo biológico y la relevancia de utilizarlo con eficiencia.

Parece existir un conflicto entre el beneficio que los encuestados perciben del uso de los animales $(89 \%$ de los estudiantes consideran que existe tal beneficio) $y$ las desventajas $u$ objeciones respecto a tal práctica (un $90 \%$ de los estudiantes cree que no hay

educativos, aunque un $89 \%$ opinó que esta práctica aporta un beneficio en su aprendizaje solo un $10 \%$ lo vieron como una actividad exclusivamente ventajosa, es decir, el $90 \%$ restante no encontró ninguna ventaja o consideró que el uso de animales es ventajoso pero también acarrea algunas desventajas.

ventajas o que existen desventajas). Aunque el uso de animales en docencia es necesario para alcanzar algunos objetivos en la enseñanza de la fisiología en las ciencias médicas [9], la existencia de una mayoría de estudiantes que en mayor o menor grado objetan su uso plantea la necesidad de que tal práctica se limite a las situaciones más justificadas, que se empleen como complemento de un proceso en el que el estudiante desarrolle destrezas que le permitan sacar el mayor provecho posible y que, en la medida que los objetivos didácticos lo permitan, se sustituya por algún otro método educativo. 
Cuadro \# 1: Percepción que tiene el estudiante de medicina de la Universidad de Costa Rica acerca del uso de animales en docencia.

\begin{tabular}{|c|c|}
\hline ITEM EVALUADO & $\begin{array}{c}\text { PORCENTAJE DE } \\
\text { ESTUDIANTES QUE } \\
\text { RESPONDIERON } \\
\text { AFIRMATIVAMENTE }\end{array}$ \\
\hline \multicolumn{2}{|l|}{$\begin{array}{l}\text { Poseen información o experiencia } \\
\text { previa sobre el uso de animales. }\end{array}$} \\
\hline $\begin{array}{l}\text { Usaron animales en cursos de } \\
\text { ciencias } \\
\text { universidad. }\end{array}$ & 98 \\
\hline $\begin{array}{l}\text { Usaron animales en secundaria. } \\
\text { Conocen regulación sobre el uso de } \\
\text { animales. }\end{array}$ & $\begin{array}{c}1 \\
73\end{array}$ \\
\hline \multicolumn{2}{|l|}{$\begin{array}{l}\text { Opinión y conocimiento sobre el uso } \\
\text { futuro de animales en docencia. }\end{array}$} \\
\hline Aporta un beneficio. & 89 \\
\hline Tiene ventajas educativas. & 10 \\
\hline $\begin{array}{l}\text { Conoce los cuidados que deben } \\
\text { darse a los animales. }\end{array}$ & 94 \\
\hline $\begin{array}{r}\begin{array}{c}\text { Conoce la procedencia de los } \\
\text { animales. }\end{array}\end{array}$ & 36 \\
\hline $\begin{array}{l}\text { Conoce el destino que tendrán los } \\
\text { animales después de usados. }\end{array}$ & 2 \\
\hline
\end{tabular}

El uso de métodos alternativos puede incluir las simulaciones por computadora. Éstas tienen la desventaja de que el estudiante se limite a seguir las instrucciones del texto de laboratorio sin preocuparse por comprender el fenómeno fisiológico o aprender sobre los principios involucrados [7]. Rendas et al. [10] utilizaron con éxito una metodología de aprendizaje basado en problemas y en mapas conceptuales para un curso de fisiopatología. Cortright et al. [11] encontraron una mejoría significativa en el aprendizaje cuando se empleó una metodología de enseñanza en pares. Taradi y Taradi [12] encontraron diferencias estadísticamente significativas en las notas obtenidas durante un curso de fisiología para estudiantes de medicina cuando estos participaron en un foro de discusión en internet junto con los profesores del curso, debido, según los autores, a que esta modalidad ayuda a superar las barreras de tiempo y espacio impuestas por un curso tradicional. Taradi et al. [13] observaron un mejor desempeño en la solución de problemas sobre equilibrio ácido-base cuando los estudiantes de un curso de fisiología participaron en un proceso de aprendizaje basado en problemas empleando un sistema de cooperación por internet. Abraham et al. [14] observaron un mejor desempeño en los exámenes de un grupo de estudiantes de fisiología después que participaron en un proceso denominado enseñanza

Revista electrónica publicada por el Departamento de Farmacología de la Escuela de Medicina de la Universidad de Costa Rica, 2060 San José, Costa Rica. ® All rights reserved. 
de fisiología orientada clínicamente el cual se basa en el planteamiento de preguntas de pensamiento crítico y en el estudio de casos clínicos.

Aunque para Costa Rica no existe información directa acerca del patrón histórico del uso de animales en docencia, si se compara el porcentaje de prácticas de los cursos fisiología humana en la Escuela de Medicina de la Universidad de Costa Rica que emplean animales se observa que este pasó de

\section{Conclusiones}

1. En una muestra de estudiantes de segundo año de la carrera de medicina en la Universidad de Costa Rica $(n=88)$ el $89 \%$ percibió como beneficioso el uso de animales en su formación profesional.

2. Un $90 \%$ de los estudiantes encuestados no encontró ninguna ventaja 0 encontró alguna desventaja en el uso de animales dentro de los cursos de la carrera.

3. Aunque un $74 \%$ de los estudiantes dice conocer la

\section{Bibliografía}

1. Lujan HL, DiCarlo SE. Too much teaching, not enough learning: what is the solution? Adv.Physiol.Educ. 2006; 30:17-22.

2. Michael J.In pursuit of meaningful learning. Adv.Physiol.Educ. 2001; 25:145-58.

3. Rothe CF, Gersting JM. Cardiovascular interactions: an interactive tutorial and mathematical model. Adv.Physiol.Educ. 2002; 26:98-109.
$41 \%$ en 1968 [15] a $14 \%$ en el 2006 [16].

Los resultados que aquí se presentan son relevantes dado que la percepción que tiene el estudiante sobre el uso de animales en docencia podría estar influyendo en su actitud hacia el aprendizaje. Este factor debería ser considerado cuidadosamente ya que propiciando una actitud abierta y cooperadora en el estudiante se podría facilitar el aprendizaje "con sentido" y con "comprensión" [17].

existencia de regulaciones respecto al uso de animales pocos (entre 2 y $36 \%$ ) conocen detalles de las mismas.

4. A partir de estos resultados y de la tendencia hacia la disminución en el uso de animales en docencia que se ha observado en otros países y parece existir en Costa Rica se sugiere el uso de métodos alternativos siempre $y$ cuando satisfagan los objetivos didácticos de los cursos.

4. McGrath $P$, Kucera $R$, Smith W. Computer simulation of introductory neurophysiology. Adv.Physiol.Educ. 2003; 27:120-9.

5. American Physiological Society. APS Position statements on animals in teaching. The Physiologist. 2005; 48(4):206-8.

6. Carrol RG. Using animals in teaching: APS position statement and rationale. The Physiologist. 2005; 48(4):206-9.

Revista electrónica publicada por el Departamento de Farmacología de la Escuela de Medicina de la Universidad de Costa Rica, 2060 San José, Costa Rica. ${ }^{\circledR}$ All rights reserved. 
7. Ra'anan AW. The evolving role of animal laboratories in physiology instruction. Adv.Physiol.Educ. 2005; 29:144-150.

8. Institute of Laboratory Animal Resources. Principles and Guidelines for the Use of Animals in Precollege Education. National Research Council, Washington. 1989.

9. Carrol RG. Design and evaluation of a national set of learning objectives: the medical physiology learning objectives project. Adv.Physiol.Educ. 2001; 25:2-7.

10. Rendas $A B$, Fonseca $M$, Rosado Pinto $P$. Toward meaningful learning in undergraduate medical education using concept maps in a PBL pathophysiology course. Adv.Physiol.Educ. 2006; 30:239.

11. Cortright RN, Collins H, DiCarlo SE. Peer instruction enhanced meaningful learning: ability to solve novel problems. Adv.Physiol.Educ. 2005; 29:107-11.

12. Taradi SK, Taradi M. Expanding the traditional physiology class with asynchronous online discussions and collaborative Adv.Physiol.Educ. 2003; 28:73-8.
13. Taradi SK, Taradi M, Radic K, Pokrajac $\mathrm{N}$. Blending problem-based learning with web technology positively impacts student learning outcomes in acid-base physiology. Adv.Physiol.Educ. 2005; 29:35-9.

14. Abraham RR, Upadhya $S$, Torke $S$, Ramnarayan K. 2004. Clinically oriented physiology teaching: strategy for developing critical-thinking skills in undergraduate medical students. Adv.Physiol.Educ. 2004; 28:102-4.

15. Gutiérrez Sáenz R. Manual de prácticas de laboratorio del curso de fisiología humana para estudiantes de medicina. Publicaciones de la Universidad de Costa Rica. Serie Ciencias Médicas No. 45. San José, Costa Rica. 1968. Pp. 210.

16. Castro R, Suárez A (Eds). Sin fecha. Prácticas de laboratorio. Fisiología Humana. Departamento de Fisiología, Universidad de Costa Rica. San José, Costa Rica. Pp. 126.

17. Michael J. Where's the evidence that active learning works? Adv.Physiol.Educ. 2006; 30:159-67.

\section{Correspondencia: Jorge Granados Zúñiga}

Departamento de Fisiología. Escuela de Medicina, Universidad de Costa Rica. Tel. 207-4485.

Correo electrónico:

jgranado@cariari.ucr.ac.cr

Revista electrónica publicada por el Departamento de Farmacología de la Escuela de Medicina de la Universidad de Costa Rica, 2060 San José, Costa Rica. (®) All rights reserved. 